5 Siyuan Fan $\mathrm{MD}^{1 *}$, Xiaojuan Wang $\mathrm{MD}^{2 *}$, Yafang $\mathrm{Hu} \mathrm{MD}, \mathrm{PhD}^{3}$, Jingping Shi MD ${ }^{4}$,

6 Yueli Zou MD ${ }^{5}$, Weili Zhao, $\mathrm{MS}^{6}$, Xiaodong Qiao $\mathrm{MS}^{6}$, Chunjuan Wang, MD ${ }^{7}$,

7 Jerome H. Chin, MD, $\mathrm{PhD}, \mathrm{MPH}^{8}$, Lei Liu MD ${ }^{9}$, Lingzhi Qin $\mathrm{MD}^{2}$, Shengnan Wang

$8 \mathrm{MD}^{3}$, Hongfang Li MB ${ }^{10}$, Wei Yue MD ${ }^{11}$, Weihe Zhang, MD ${ }^{12}$, Xiaohua Li MM ${ }^{13}$,

9 Ying Ge $\mathrm{MD}^{14}$, Honglong Wu MS ${ }^{15,16}$, Weijun Chen $\mathrm{PhD}^{17,18}$, Yongjun $\mathrm{Li} \mathrm{PhD}^{19}$,

10

\section{Metagenomic Next-generation Sequencing of Cerebrospinal Fluid for the}

\section{Diagnosis of Central Nervous System Infections: A Multicentre Prospective}

\section{Study}

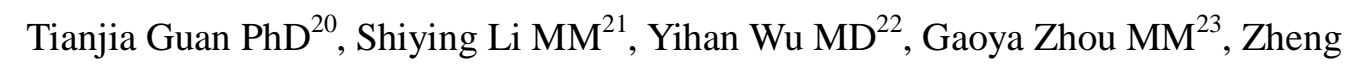

Liu $\mathrm{MD}^{24}$, Yushun Piao MD ${ }^{25}$, Jianzhao Zhang $\mathrm{MM}^{26}$, Changhong Ren $\mathrm{MD}^{27}$, Li Cui,

MD, $\mathrm{PhD}^{28}$, Caiyun Liu MD ${ }^{28}$. Haitao Ren $\mathrm{BS}^{1}$, Yanhuan Zhao $\mathrm{BS}^{1}$, Shuo Feng $\mathrm{MM}^{26}$,

Haishan Jiang $\mathrm{MD}^{3}$, Jiawei Wang $\mathrm{MD}^{9}$, Hui Bu MD , Shougang Guo MD , Bin Peng

$\mathrm{MD}^{1}$, Liying Cui MD${ }^{1}$, Wei Li $\mathrm{PhD}^{2}$, Hongzhi Guan MD .

\section{${ }^{1}$ Department of Neurology, Peking Union Medical College Hospital, Chinese}

Academy of Medical Sciences and Peking Union Medical College, Beijing, China.

${ }^{2}$ Department of Neurology, People's Hospital of Zhengzhou University, Henan

Provincial People's Hospital, Zhengzhou, China.

${ }^{3}$ Department of Neurology, Nanfang Hospital, Southern Medical University,

Guangzhou, China.

${ }^{4}$ Department of Neurology, Affiliated Nanjing Hospital of Nanjing Medical 
$24{ }^{5}$ Department of Neurology, The Second Hospital of Hebei Medical University,

25 Shijiazhuang, China.

$26{ }^{6}$ Department of Neurology, Affiliated Hospital of Chifeng University, Chifeng,

27 China.

$28{ }^{7}$ Department of Neurology, Shandong Provincial Hospital Affiliated to Shandong

29 University, Jinan, China.

$30{ }^{8}$ Department of Neurology, Division of Global Health, New York University Langone

31 Health, New York, The United States of America.

$32{ }^{9}$ Department of Neurology, Beijing Tongren Hospital, Capital Medical University,

33 Beijing, China.

$34{ }^{10}$ Department of Neurology, Affiliated Hospital of Jining Medical University, Jining,

35 China.

$36{ }^{11}$ Department of Neurology, Tianjin Huanhu Hospital, Tianjin, China.

$37{ }^{12}$ Department of Neurology, China-Japan Friendship Hospital, Beijing, China.

$38{ }^{13}$ Department of Paediatrics, Affiliated Hospital of Inner Mongolia Medical

39 University, Hohhot, China.

$40{ }^{14}$ Department of Infectious Diseases, Peking Union Medical College Hospital,

41 Chinese Academy of Medical Sciences and Peking Union Medical College, Beijing,

42 China.

$43 \quad{ }^{15}$ Tianjin Medical Laboratory, BGI-Tianjin, BGI-Shenzhen, Tianjin, China.

$44{ }^{16}$ Binhai Genomics Institute, BGI-Tianjin, BGI-Shenzhen, Tianjin, China. 
45

46

47

48

49

50

51

52

53

54

55

56

57

58

59

60

61

62

63

64

65

66
${ }^{17}$ University of Chinese Academy of Sciences, Beijing, China.

${ }^{18}$ Key Laboratory of Genome Sciences and Information, Beijing Institute of

Genomics, Chinese Academy of Sciences, Beijing, China.

${ }^{19}$ BGI genomics, BGI-Shenzhen, Shenzhen, China.

${ }^{20}$ School of Public Health, Chinese Academy of Medical Sciences and Peking Union

Medical College, Beijing, China.

${ }^{21}$ Department of Neurology, North China University of Science and Technology,

Tangshan, China.

${ }^{22}$ Department of Neurology, Inner Mongolia People's Hospital, Hohhot, China.

${ }^{23}$ Department of Neurology, Brain Hospital of Hunan Province, Changsha, China.

${ }^{24}$ Department of Neurology, Xuanwu Hospital, Capital Medical University, Beijing,

China.

${ }^{25}$ Department of Neurology, Shenzhen Baoan Shiyan People's Hospital, Shenzhen,

China.

${ }^{26}$ Department of Neurology, Capital Institute of Paediatrics, Beijing, China.

${ }^{27}$ Department of Neurology, Beijing Children's Hospital, Capital Medical University,

Beijing, China.

${ }^{28}$ Department of Neurology and Neuroscience Centre, the First Hospital of Jilin

University, Changchun, China.

* Siyuan Fan and Xiaojuan Wang have contributed equally to this work. 
67 Corresponding author: Hongzhi Guan.

68 Department of Neurology, Peking Union Medical College Hospital, Chinese Academy

69 of Medical Sciences and Peking Union Medical College, Beijing 100730, China

70 Telephone Number: (0086) 10-69156371

71 Fax Number: (0086) 10-69156371

72 Email: guanhz@263.net

73

74 Corresponding author: Wei Li.

75 Department of Neurology, People's Hospital of Zhengzhou University, Henan

76 Provincial People's Hospital, Zhengzhou 450003, China.

77 Telephone Number: (0086) 13939061069

78 Fax Number: (0086) 371-65897739

79 Email: $\underline{\text { liwein@ zzu.edu.cn }}$

80

81 Word Count: 2872

82 


\section{ABSTRACT}

86 Background: Infectious encephalitis and meningitis are often treated empirically

87 without identification of the causative pathogen. Metagenomic next-generation

88 sequencing (mNGS) is a high throughput technology that enables the detection of

89 pathogens independent of prior clinical or laboratory information.

90 Methods: The present study was a multicentre prospective evaluation of mNGS of

91 cerebrospinal fluid (CSF) for the diagnosis of suspected central nervous system

92 infections.

93 Results: A total of 276 patients were enrolled in this study between Jan 1, 2017 and

94 Jan 1, 2018. Identification of an etiologic pathogen in CSF by mNGS was achieved in

95101 patients $(36.6 \%)$. mNGS detected 11 bacterial species, 7 viral species, 2 fungal

96 species, and 2 parasitic species. The five leading positive detections were

97 varicella-zoster virus (17), Mycobacterium tuberculosis (14), herpes simplex virus 1

98 (12), Epstein-Barr virus (12), and Cryptococcus neoformans (7). False positives

99 occurred in $12(4.3 \%)$ patients with bacterial infections known to be widespread in

100 hospital environments. False negatives occurred in $16(5.8 \%)$ patients and included

101 bacterial, viral and fungal aetiologies.

102 Conclusions: mNGS of CSF is a powerful diagnostic method to identify the pathogen

103 for many central nervous system infections.

105 Keywords: encephalitis, meningitis, metagenomic next-generation sequencing,

106 diagnosis 
107

108

109

110

111

112

113

114

115

\section{INTRODUCTION}

Infectious encephalitis and meningitis are major contributors to the neurological

global burden of disease ${ }^{1-4}$. Numerous microorganisms, including bacteria, viruses, fungi, and parasites, can cause encephalitis and meningitis in immunocompetent or immunocompromised hosts; but the clinical manifestations of many infections are non-specific. Using comprehensive conventional diagnostic technologies, microbiological detection of the pathogen is achieved in only $50-80 \%$ of cases $^{5-8}$. The inability to identify the infectious aetiology of encephalitis and meningitis often results in delayed, inadequate, and/or inappropriate treatment.

Metagenomic next-generation sequencing (mNGS) is a novel tool that allows for the simultaneous and independent sequencing of thousands to billions of DNA fragments ${ }^{9}$. Cerebrospinal fluid (CSF) is particularly suitable for NGS due to its sterility in healthy individuals. Compared with traditional individual target-specific tests, mNGS can identify pathogens without the input of clinical predictors or prior laboratory results. Several recent studies have demonstrated the capability of mNGS of CSF to identify known and unsuspected pathogens and to discover new microorganisms ${ }^{10-18}$. mNGS of CSF is being increasingly utilized in routine clinical settings for the rapid diagnosis of central nervous system infections. However, most published studies are retrospective case reports or case series ${ }^{11-17,19-24}$, and thus, large prospective studies are needed to demonstrate the clinical impact and cost-effectiveness of mNGS for the diagnosis of meningitis and encephalitis. We undertook a multicentre prospective study to comprehensively evaluate the 
129

130

131

132

133

134

135

136

137

performance of mNGS of CSF for the diagnosis of central nervous system (CNS)

infections compared to conventional microbiological methodologies.

\section{METHODS}

\subsection{Participants and study design}

This study was a multicentre prospective cohort assessment of the mNGS of CSF for the diagnosis of suspected infectious encephalitis or meningitis. The participating sites were 20 hospitals located in 10 provinces/municipalities in China. Each hospital is a member of the Beijing Encephalitis Group. Adult patients were eligible for inclusion in the study if they presented with clinical manifestations consistent with either encephalitis or meningitis (Table 1) and if standard diagnostic examinations (Supplementary Table 1) failed to identify an etiological cause within 3 days. Exclusion criteria are shown in Table 1.

mNGS were conducted on all CSF specimens. Relevant conventional microbiological studies (e.g. staining, culture, polymerase chain reaction [PCR], serology) were arranged according to the clinical manifestations and the results of mNGS. Conventional microbiological studies were considered the gold standard according to relevant guidelines and/or consensus ${ }^{2,25-27}$ to classify the results of mNGS as true-positive, false-positive, and false-negative. Detected pathogens were classified as etiologic pathogens if the major clinical manifestations of the patient were consistent with that pathogen. All patients were treated based on the results of conventional microbiological testing (or empirically if results were negative) 
151

152

153

154

155

156

157

158

159

160

161

162

163

164

165

166

167

168

169

170

171

172

according to the latest clinical guidelines and/or consensus. Patients were followed for

at least 30 days to determine the final diagnosis. Demographic data, medical history,

laboratory test results (including all conventional microbiological tests),

neuroimaging findings, medical therapy, and response to treatment were collected

prospectively. Patients enrolled from Jan 1, 2017 to Jan 1, 2018 were included in the

final analyses.

This study was approved by the institutional review board of Peking Union

Medical College Hospital (no. JS-890). Written informed consent was obtained from each patient or their legal surrogate prior to enrolment.

\section{2 mNGS of CSF}

CSF samples were collected according to standard sterile procedures, snap-frozen, and stored at $-20^{\circ} \mathrm{C}$ until they were delivered to the sequencing centre. Because

reverse transcription was not performed to prepare DNA libraries, RNA viruses were not investigated in this study. mNGS of the CSF samples was performed using a standard flow that has been successfully used to detect herpes simplex virus 1 (HSV1), HSV2, varicella zoster virus (VZV), Listeria monocytogenes, Brucella, and Taenia solium $^{12-15}$.

DNA was extracted from $300 \mu \mathrm{L}$ of CSF and negative 'no-template' controls (NTCs). Sequencing was performed on the BGISEQ-100 platform with an average of 20 million total reads obtained for each sample. The qualified reads were mapped to the human reference genome using the Burrows-Wheeler Aligner to remove human 
173

sequences. The remaining reads were aligned to the database of annotation, which includes the NCBI microbial genome database (ftp://ftp.ncbi.nlm.nih.gov/genomes/)

to detect pathogens. The sequencing data was analysed in terms of the numbers of raw reads, non-human reads, and reads aligned to the microbial genome database as well as species-specific reads (genus-specific reads for Mycobacterium tuberculosis and Brucella), reads per million (RPM), and genome coverage (\%). The results of mNGS were available in less than $48 \mathrm{hrs}$.

\subsection{Criteria for positive results of mNGS of CSF samples}

To reduce the influence of potential contamination, we used the following criteria for positive results of CSF mNGS:

1) For extracellular bacteria, fungi (excluding Cryptococcus), and parasites, the result was considered positive if a species detected by mNGS had a species-specific read number $(\mathrm{SSRN}) \geq 30(\mathrm{RPM} \geq 1.50)$ that ranked among the top 10 for bacteria, fungi, or parasites. Organisms detected in the NTC or that were present in $\geq 25 \%$ of samples from the previous 30 days were excluded but only if the detected SSRN was $\geq 10$-fold than that in the $\mathrm{NTC}^{28}$ or other organisms. Additionally, organisms present in $\geq 75 \%$ of samples from the previous 30 days were excluded.

2) For intracellular bacteria (excluding Mycobacterium tuberculosis and Brucella) and Cryptococcus, the result was considered positive if a species detected by NGS had a $S S R N \geq 10(\mathrm{RPM} \geq 0.50)^{13}$ that ranked among the top 10 for bacteria or fungi. Pathogens detected in the NTC or that were present in $\geq 25 \%$ of samples from the 
195

previous 30 days were excluded but only if the detected SSRN was $\geq 10$-fold than that in the NTC or other organisms.

considered positive if a species (or genus for Mycobacterium tuberculosis

[Mycobacterium tuberculosis complex, MTC] and Brucella) detected by NGS had a

SSRN $\geq 3(R P M \geq 0.15)^{12,28}$. Pathogens detected in the NTC were excluded but only

if the detected SSRN was $\geq 10$-fold than that in the NTC. In our previous clinical

observations, there were a few cases without Mycobacterium tuberculosis infection

which contained MTC-specific reads number of 1 in the mNGS results. To mitigate

the possibility of false positives, we adopted the criteria of SSRN $\geq 3$ rather than

$\mathrm{SSRN} \geq 1^{24}$ in this study.

The performance of the criteria were evaluated at the finally stage of the study,

the original results of mNGS and/or clinical manifestations were used to guide the further testing of conventional microbiological studies.

\subsection{Statistical analysis}

All statistical analyses were conducted using Statistical Package for the Social Sciences (SPSS) version 17.0 and EXCEL 1810. Depending on their distribution, all data are expressed as medians with interquartile ranges (IQRs) or as means \pm standard deviation.

\section{RESULTS}




\section{$217 \quad 3.1$ Characteristics of the study participants}

218287 patients were screened for inclusion in this study (Fig. 1). 11 patients were

219 initially thought to have CNS infections and mNGS was performed. However, these

220 cases were ultimately excluded following the final diagnosis of a non-infectious

221 disease. Of these 11 excluded patients, 10 had negative mNGS results, and 1 patient

222 receiving immunosuppressive therapy was positive for BK polyomavirus. The final

223 cohort included 276 patients in the study. $176(63.8 \%)$ were male and the median age

224 was 42 years (IQR: 26-54 years). The median time from disease-onset to CSF

sampling was 10 days (IQR: 5-25 days). The median white blood cell count in CSF

was $80 / \mathrm{mm}^{3}$ (IQR: $19-220 / \mathrm{mm}^{3}$ ). The median CSF monocyte cell count was $36 / \mathrm{mm}^{3}$

227 (IQR: $10-127 / \mathrm{mm}^{3}$ ). During a follow-up period of 30 days, nine patients died.

\subsection{Performance of mNGS for diagnosing CNS infections}

276 CSF samples were tested by mNGS and conventional microbiological studies. 122

samples were positive by mNGS (110 true positive, 12 false positive), 126 were

positive by conventional microbiological tests, and 114 total positive results were

considered "Etiologic Pathogens" (Table 2). All mNGS results were obtained in less

microbiological tests were positive.

Of the patients first diagnosed by mNGS, $16.3 \%$ of infections were caused by

In total, NGS detected 11 bacterial species, of which M. tuberculosis (14 cases, 
239

240

241

242

243

244

245

246

247

248

249

250

251

252

253

254

255

256

257

258

259

260

$13.9 \%$ ) and L. monocytogenes (7.9\%) were the most common (Fig. 2B), 7 viral

species (BK polyomavirus was not the etiologic pathogen), of which VZV (16.8\%)

and HSV1 (11.9\%) were the most common, 2 fungal species, both of which were

Cryptococcus (7.9\%), and 2 parasitic species, of which $T$. solium (5.0\%) was the most

common. Nine co-infections with Epstein-Barr virus (EBV) (three with HSV1, two

with Brucella, one with Cryptococcus, one with S. haemolyticus, one with $P$.

aeruginosa, and one with $M$. tuberculosis), two co-infections with cytomegalovirus

(CMV) (one with M. tuberculosis, and one with Cryptococcus), and one co-infection

with BK polyomavirus (with HSV1) were detected. The EBV and BK polyomavirus

did not appear to be consistent with the clinical manifestations in these two instances

of co-infections.

\subsection{False positive results of CSF mNGS}

In the present study, false positives occurred in $12(4.3 \%)$ patients and were primarily associated with bacterial infections (n=12; Table 2), including E. coli, E. faecium, A.

baumannii, S. maltophilia, and P. aeruginosa, and a false positive for Brucella was

also seen. Of note, the false-positive samples contained numerous other bacteria, that

could be detected simultaneously by NGS. Using our proposed criteria, there were no

false positives for viruses, fungi, or parasites. Although EBV was not the etiologic

pathogen in most cases, it was present in the CSF of some patients. Additionally, there

was some background contamination in most CSF samples (Supplementary Table 2)

but these organisms did not meet the criteria for a positive result. 


\subsection{False negative results of CSF mNGS}

263 In the present study, false negatives occurred in $16(5.8 \%)$ patients (Table 2$)$ and were

264 associated with bacterial, viral and fungal infections. The false negative cases of

265 bacterial infection were all treated with antibiotics prior to sequencing. In the false

266 negative cases of viral infection, 1 or 2 SSRNs were detected in the samples but did

267 not satisfy the proposed criteria for a positive $\mathrm{mNGS}$ result. If the criteria for a

268 positive result was relaxed to a $S S R N \geq 1(R P M \geq 0.05)$, there were no false negative

269 cases of HSV1 or VZV or false positive cases of HSV1, HSV2 or VZV. In this study,

270 if we adopted the alternate criteria $S S R N \geq 1(R P M \geq 0.05)$ for viruses and

271 Mycobacterium tuberculosis, there would be additional potential false positives,

272 including $30 \mathrm{EBV}, 7 \mathrm{CMV}$ and 5 Mycobacterium tuberculosis infections. It should be

273 pointed out that the possibility of Mycobacterium tuberculosis infection in the 5 cases

274 cannot be ruled out based on the clinical and paraclinical manifestations, because the

275 conventional microbiological methods might fail to detect the Mycobacterium

276 tuberculosis. Of note, there were three false negative cases of Cryptococcus infection.

\section{4. DISCUSSION}

279 To the best of our knowledge, the present study is the first to assess the performance

280 of mNGS for pathogen identification in a large prospective cohort of patients with

281 suspected CNS infections. Specifically, our study compared results of mNGS of CSF

282 to conventional microbiological studies and proposed new criteria for validating a 
283

284

285

286

287

288

289

290

291

292

293

294

295

296

297

298

299

300

301

302

303

304

mNGS result as positive for therapeutic decision-making. Our results suggest that

NGS can provide a quicker and more accurate etiologic pathogen identification than conventional microbiological methods. However, patients in the present study were only eligible to be assessed by mNGS if conventional microbiological studies, e.g. routine bacterial stains and cultures, India ink preparation, targeted PCR tests, serological tests, failed to identify an etiologic cause within 3 days. Thus, the application of CSF mNGS in the clinical setting of this study could be regarded as a quasi-first line method for diagnosing CNS infectious diseases.

mNGS is a high-throughput sequencing technique without the requirement of prior information, allowing detection of unsuspected or novel organisms. Importantly, mNGS can detect unsuspected pathogens that clinicians may fail to consider because of atypical clinical manifestations. Many cases of neurological infections have been unexpectedly diagnosed by mNGS of $\mathrm{CSF}^{11,22,29,30}$ similar to the present study for the cases of L. monocytogenes, Brucella and T. solium ${ }^{12-14}$. In addition, as demonstrated in previous studies ${ }^{10,20,21}$ and in the present study for the case of encephalitis caused by Suid herpesvirus $1^{31}$, mNGS of CSF has the ability to identify novel aetiologies of CNS infections. Furthermore, NGS can detect unexpected co-infections that may guide appropriate targeted treatment. For example, we detected co-infections of CMV and Cryptococcus. In routine clinical practice, if conventional microbiological methods detect Cryptococcus, then no further tests for other microorganisms other than HIV are usually performed. Finally, mNGS of CSF may be an appropriate tool for ruling out a broad spectrum of potential CNS infectious diseases prior to 
305

306

307

308

309

310

311

312

313

314

315

316

317

318

319

320

321

322

323

324

325

326

concluding a final diagnosis of autoimmune diseases, such as autoantibody-negative autoimmune encephalitis.

Contamination of samples during specimen collection and/or processing is a major challenge when interpreting mNGS results. To reduce the potential influence of contamination, we defined strict criteria for positive mNGS results. The various types of contamination observed in the present study could be divided into two groups: (1) microorganisms commonly associated with background contamination that did not meet the criteria for a positive result (Supplementary Table 2) and (2) false positive detections that fulfilled our criteria for a positive mNGS result but were not consistent with the patients' clinical presentation and features. The contaminations derived primarily from the following sources: (1) laboratory practices (Parvovirus NIH CQV is a contaminant from silica column-based nucleic acid extraction kits $)^{32}$; (2) reagents (Bradyrhizobium, Burkholderia and Ralstonia are common contaminants used in industrial ultrapure water systems $)^{33,34}$; (3) environment (E. coli, P. aeruginosa, E. faecium and Torque teno virus are widespread pathogens in hospital environment) ${ }^{35,36}$; (4) skin or other body flora (P. acnes, M. globose, E. coli and S. epidermidis are widely associated with the human skin flora) ${ }^{37,38}$. False positive results are very likely to misguide treatment, and therefore, clinicians should be cautious when interpreting positive mNGS detection of extracellular bacteria or fungi that are widespread in hospital environments, especially when many species of bacteria are detected in a single NGS test. On the other hand, positive mNGS detection of viruses and parasites are not likely to be false positives. 
334 lower limit of detection for nucleic acid amplification tests ${ }^{39,40}$. The criteria of SSRN

$335 \geq 3$ rather than $\mathrm{SSRN} \geq 1$ might introduce false-negative cases for Mycobacterium the specified criteria for a positive result.

first-line laboratory method in the response to emerging infectious diseases and

347 decision-making. mNGS of CSF should be considered as a first-line test for acute 
certain pathogen does not necessarily prove that it is responsible for the patient's

clinical presentation and features ${ }^{23,27}$. In the present study, some patients were positive

detect microorganisms that are not included in microbial genome databases.

Next-generation RNA sequencing should be performed in future prospective studies. 


\section{$371 \quad$ List of abbreviations}

372 mNGS: metagenomic next-generation sequencing

373 CSF: cerebrospinal fluid

374 CNS: central nervous system

375 PCR: polymerase chain reaction

376 HSV: herpes simplex virus

377 VZV: varicella zoster virus

378 NTC: 'no-template' controls

379 RPM: reads per million

380 EBV: Epstein-Barr virus

381 CMV: cytomegalovirus

382 SSRN: species-specific read number

\section{Declarations}

385 Ethics approval and consent to participate

386 This study was approved by the institutional review board of Peking Union Medical

387 College Hospital (no. JS-890). Written informed consent was obtained from each

388 patient or their legal surrogate prior to enrolment.

\section{Consent for publication}

390 Not applicable.

\section{$391 \quad$ Availability of data and materials}

392 The datasets used and/or analysed during the current study are available from the 
393

394

395

396

397

398

399

400

401

402

403

404

405

406

407

408

409

410

411

412

413

corresponding author on reasonable request.

\section{Competing interests}

Author Honglong Wu was employed by company BGI-Tianjin and BGI-Shenzhen.

Author Yongjun Li was employed by company BGI-Shenzhen. All other authors declare no competing interests.

\section{Funding}

This study was funded by the National Key Research and Development Program of China (Grant No. 2016YFC0901500); and National Science and Technology Major Project of China (Grant No.2018ZX10305409)

\section{Author's contributions}

HG and SF contributed to the study conception design. All the authors participated in the discussion of the study design. SF, XW, YF, JS, YZ, WZ, XQ, CW, LL, LQ, SW, HJ, HL, WY, WZ, XL, SL, YW, YP, HR, YZ, JW, BP, LC, WL, and HG enrolled patients and collected the clinical data. HW, YL and WC performed NGS, bioinformatics analysis, and PCR. SF, HG, XW, and WL analyzed the data. SF wrote the first draft of the manuscript after discussions with HG, XW and WL. HW wrote portions of the Methods section. HG, JHC, TG and WC contributed to manuscript revision. All authors have read and approved the submitted version.

\section{Acknowledgments}

The authors thank the patients for participating in this study. 


\section{REFERENCE}

415 1. McGill F, Heyderman RS, Panagiotou S, Tunkel AR, Solomon T: Acute 416 bacterial meningitis in adults. Lancet 2016, 388(10063):3036-3047.

417 2. Venkatesan A, Tunkel AR, Bloch KC, Lauring AS, Sejvar J, Bitnun A, Stahl JP, 418 Mailles A, Drebot M, Rupprecht CE et al: Case definitions, diagnostic 419 algorithms, and priorities in encephalitis: consensus statement of the international encephalitis consortium. Clin Infect Dis 2013, 57(8):1114-1128.

3. Feigin VL, Nichols E, Alam T, Bannick MS, Beghi E, Blake N, et al: Global, regional, and national burden of neurological disorders, 1990-2016: a

online March 14, 2019]. Lancet Neurol. doi: 10.1016/S1474-4422(18)30499-X.

4. Feigin VL, Abajobir AA, Abate KH, Abd-Allah F, Abdulle AM, Abera SF, et al: Global, regional, and national burden of neurological disorders during 1990-2015: a systematic analysis for the Global Burden of Disease Study 2015. Lancet Neurol. 2017;16(11):877-897.

5. Khatib U, van de Beek D, Lees JA, Brouwer MC. Adults with suspected central nervous system infection: A prospective study of diagnostic accuracy. $J$ Infect. 2017;74(1):1-9.

6. Hasbun R, Rosenthal N, Balada-Llasat JM, Chung J, Duff S, Bozzette S, United States, 2011-2014. Clin Infect Dis. 2017;65(3):359-363. 
7. Granerod J, Ambrose HE, Davies NW, Clewley JP, Walsh AL, Morgan D, Cunningham R, Zuckerman M, Mutton KJ, Solomon $\mathrm{T}$ et al: Causes of encephalitis and differences in their clinical presentations in England: a multicentre, population-based prospective study. Lancet Infect Dis. 2010;10(12):835-844.

8. Glaser CA, Honarmand S, Anderson LJ, Schnurr DP, Forghani B, Cossen CK, Schuster FL, Christie LJ, Tureen JH: Beyond viruses: clinical profiles and etiologies associated with encephalitis. Clin Infect Dis. 2006;43(12):1565-1577.

9. Gu W, Miller S, Chiu CY. Clinical Metagenomic Next-Generation Sequencing for Pathogen Detection. Annu Rev Pathol. 2019;24(14):319-338.

10. Wilson MR, Suan D, Duggins A, Schubert RD, Khan LM, Sample HA, Zorn KC, Rodrigues Hoffman A, Blick A, Shingde M et al: A Novel Cause of Chronic Viral Meningoencephalitis: Cache Valley Virus. Ann Neurol. 2017;82(1):105-114.

11. Mongkolrattanothai K, Naccache SN, Bender JM, Samayoa E, Pham E, Yu G, Dien Bard J, Miller S, Aldrovandi G, Chiu CY: Neurobrucellosis: Unexpected Answer From Metagenomic Next-Generation Sequencing. J Pediatric Infect Dis Soc. 2017;6(4):393-398.

12. Fan S, Ren H, Wei Y, Mao C, Ma Z, Zhang L, Wang L, Ge Y, Li T, Cui L et al: Next-generation sequencing of the cerebrospinal fluid in the diagnosis of neurobrucellosis. Int J Infect Dis. 2018;67:20-24. 
458

459

460

461

462

463

464

465

466

467

468

469

470

471

472

473

474

475

476

477

478

479

13. Yao M, Zhou J, Zhu Y, Zhang Y, Lv X, Sun R, Shen A, Ren H, Cui L, Guan H et al: Detection of Listeria monocytogenes in CSF from Three Patients with Meningoencephalitis by Next-Generation Sequencing. $J$ Clin Neurol. 2016;12(4):446-451.

14. Fan S, Qiao X, Liu L, Wu H, Zhou J, Sun R, Chen Q, Huang Y, Mao C, Yuan J et al: Next-Generation Sequencing of Cerebrospinal Fluid for the Diagnosis of Neurocysticercosis. Front Neurol. 2018;9:471.

15. Guan H, Shen A, Lv X, Yang X, Ren H, Zhao Y, Zhang Y, Gong Y, Ni P, Wu H et al: Detection of virus in CSF from the cases with meningoencephalitis by next-generation sequencing. J Neurovirol. 2016;22(2):240-245.

16. Wilson MR, Shanbhag NM, Reid MJ, Singhal NS, Gelfand JM, Sample HA, Benkli B, O'Donovan BD, Ali IK, Keating MK et al: Diagnosing Balamuthia mandrillaris Encephalitis With Metagenomic Deep Sequencing. Ann Neurol. 2015;78(5):722-730.

17. Wilson MR, Naccache SN, Samayoa E, Biagtan M, Bashir H, Yu G, Salamat SM, Somasekar S, Federman S, Miller S et al: Actionable diagnosis of neuroleptospirosis by next-generation sequencing. $N$ Engl $J$ Med. 2014;370(25):2408-2417.

18. Parize P, Muth E, Richaud C, Gratigny M, Pilmis B, Lamamy A, Mainardi JL, Cheval J, de Visser L, Jagorel $\mathrm{F}$ et al: Untargeted next-generation sequencing-based first-line diagnosis of infection in immunocompromised adults: a multicentre, blinded, prospective study. Clin Microbiol Infect. 
19. Naccache SN, Peggs KS, Mattes FM, Phadke R, Garson JA, Grant P, Samayoa infection in an immunocompromised adult with encephalitis by unbiased next-generation sequencing. Clin Infect Dis. 2015;60(6):919-923. Squirrel Bornavirus Associated with Fatal Human Encephalitis. $N$ Engl J Med. 2015;373(2):154-162.

21. Brown JR, Morfopoulou S, Hubb J, Emmett WA, Ip W, Shah D, Brooks T,

Paine SM, Anderson G, Virasami A et al: Astrovirus VA1/HMO-C: an

22. Wilson MR, O'Donovan BD, Gelfand JM, Sample HA, Chow FC, Betjemann increasingly recognized neurotropic pathogen in immunocompromised

23. Brown JR, Bharucha T, Breuer J: Encephalitis diagnosis using metagenomics: Neurol. 2018;75(8):947-955. 
504 25. Solomon T, Michael BD, Smith PE, Sanderson F, Davies NW, Hart IJ, Holland encephalitis in adults--Association of British Neurologists and British Infection Association National Guidelines. J infect. 2012;64(4):347-373. clinical practice guidelines by the Infectious Diseases Society of America. Clin Infect Dis. 2008;47(3):303-327.

27. Granerod J, Cunningham R, Zuckerman M, Mutton K, Davies NW, Walsh AL, Ward KN, Hilton DA, Ambrose HE, Clewley JP et al: Causality in acute encephalitis: defining aetiologies. Epidemiol Infect. 2010;138(6):783-800. Metagenomic Next-Generation Sequencing Tests for Universal Pathogen Detection. Arch Pathol Lab Med. 2017;141(6):776-786. Lipowski D, Pollak A, Lechowicz U, Caraballo Cortes $\mathrm{K}$ et al: Next-generation sequencing (NGS) in the identification of encephalitis-causing viruses: Unexpected detection of human herpesvirus 1 while searching for RNA pathogens. J Virol Methods. 2015;226:1-6.

523 30. Beck ES, Ramachandran PS, Khan LM, Sample HA, Zorn KC, O'Connell EM, 
Nash T, Reich DS, Venkatesan A, DeRisi JL et al: Clinicopathology conference: 41-year-old woman with chronic relapsing meningitis. Ann Neurol. 2019;85(2):161-169.

31. Zhao W, Wu Y, Li H, Li S, Fan S, Wu H, Li Y, Lu Y, Han J, Zhang W et al: Clinical experience and next-generation sequencing analysis of encephalitis caused by pseudorabies virus. National Medical Journal of China. 2018;98(15):6.

32. Strong MJ, Xu G, Morici L, Splinter Bon-Durant S, Baddoo M, Lin Z, Fewell C, Taylor CM, Flemington EK: Microbial contamination in next generation sequencing: implications for sequence-based analysis of clinical samples. PLoS Pathog. 2014;10(11):e1004437.

33. Laurence M, Hatzis C, Brash DE: Common contaminants in next-generation sequencing that hinder discovery of low-abundance microbes. PLoS One. 2014;9(5):e97876.

34. Kulakov LA, McAlister MB, Ogden KL, Larkin MJ, O'Hanlon JF: Analysis of bacteria contaminating ultrapure water in industrial systems. Appl Environ Microbiol. 2002;68(4):1548-1555.

35. Hocquet D, Muller A, Bertrand X: What happens in hospitals does not stay in hospitals: antibiotic-resistant bacteria in hospital wastewater systems. $J$ Hosp Infect. 2016;93(4):395-402.

36. Verani M, Bigazzi R, Carducci A: Viral contamination of aerosol and surfaces through toilet use in health care and other settings. Am J Infect Control. 
547 37. Lusk RW: Diverse and widespread contamination evident in the unmapped depths of high throughput sequencing data. PLoS One. 2014;9(10):e110808.

38. Nguyen TH, Park MD, Otto M: Host Response to Staphylococcus epidermidis Colonization and Infections. Front Cell Infect Microbiol. 2017;7:90. 2018;24(7):791-792.

40. Ellis JE, Missan DS, Shabilla M, Martinez D, Fry SE: Rapid infectious disease identification by next-generation DNA sequencing. J Microbiol Methods. 2017;138:12-19.

41. Grad YH, Lipsitch M, Feldgarden M, Arachchi HM, Cerqueira GC, Fitzgerald U S A. 2012;109(8):3065-3070. the new age of genomic epidemiology. Am J Infect Control. 2017;45(2):170-179. 
bioRxiv preprint doi: https://doi.org/10.1101/658047; this version posted June 10, 2019. The copyright holder for this preprint (which was not certified by peer review) is the author/funder, who has granted bioRxiv a license to display the preprint in perpetuity. It is made available under aCC-BY-NC-ND 4.0 International license. 
Table 1. Case definitions and exclusion criteria for encephalitis and meningitis

Case definitions for encephalitis

Major criteria (required)

Patients presenting to medical attention with altered mental status (decreased or altered level of consciousness or personality change) lasting $\geq 24$ hrs with no alternative cause identified, and/or generalized or partial seizures not fully attributable to a pre-existing seizure disorder or a simple febrile seizure.

Minor criteria ( $\geq 2$ points)

Documented fever $\geq 38 \square$ within the 72 hrs before or after presentation

New onset of focal neurologic findings

CSF WBC count $\geq 5 /$ cubic mm

Abnormality of brain parenchyma on neuroimaging suggestive of encephalitis that is either new from prior studies or appears acute in onset

Abnormality on EEG that is consistent with encephalitis and not attributable to another cause.

\section{Exclusion criteria for encephalitis}

$\leq 28$ days of age

Non-infectious encephalitis, such as autoimmune disorders, paraneoplastic

syndromes, NMOSD, neuropsychiatric involvement of rheumatic diseases

HIV or syphilis infection

History of recent (within 4 weeks before the onset of disease) vaccination

Meningitis without clinical brain parenchyma involvement

Absolute contraindications for lumbar puncture;

Traumatic LP with obvious blood-contaminated CSF

Pregnancy

Refusal to sign the informed consent

\section{Case definitions for meningitis}

Patients presenting to medical attention with at least two of the four symptoms of headache, fever (documented fever $\geq 38 \square$ within the $72 \mathrm{hrs}$ before or after presentation), neck stiffness, decreased level of consciousness (defined by a Glasgow

Coma Scale score below 14)

CSF white blood cell count $\geq 5 /$ cubic $\mathrm{mm}$

\section{Exclusion criteria for meningitis}

$\leq 28$ days of age

HIV or syphilis infection

Meningeal malignancy confirmed by CSF cytology

Traumatic LP with obvious blood-contaminated CSF

Pregnancy

Refusal to sign the informed consent 
Table 2. Performance of mNGS of CSF compared to conventional microbiological

571 studies (gold standard) for the diagnosis of CNS infections

\begin{tabular}{|c|c|c|c|c|}
\hline & $\mathbf{T P}^{*}$ & FP & FN & Etiologic Pathogen \\
\hline \multicolumn{5}{|l|}{ Bacteria } \\
\hline Mycobacterium tuberculosis & 14 & 0 & 1 & 15 \\
\hline Listeria monocytogenes & 8 & 0 & 0 & 8 \\
\hline Brucella & 7 & 1 & 1 & 8 \\
\hline Streptococcus pneumoniae & 5 & 0 & 0 & 5 \\
\hline Klebsiella pneumoniae & 3 & 0 & 0 & 3 \\
\hline Streptococcus intermedius & 2 & 0 & 0 & 2 \\
\hline Haemophilus influenzae & 2 & 0 & 0 & 2 \\
\hline Vibrio vulnificus & 1 & 0 & 0 & 1 \\
\hline Staphylococcus hominis & 1 & 0 & 0 & 1 \\
\hline Escherichia coli & 0 & 2 & 0 & 0 \\
\hline Enterococcus faecium & 0 & 2 & 0 & 0 \\
\hline Acinetobacter baumannii & 1 & 2 & 0 & 1 \\
\hline Stenotrophomonas maltophilia & 1 & 1 & 0 & 1 \\
\hline Pseudomonas aeruginosa & 0 & 4 & 1 & 1 \\
\hline Staphylococcus aureus & 0 & 0 & 1 & 1 \\
\hline Staphylococcus haemolyticus & 0 & 0 & 1 & 1 \\
\hline \multicolumn{5}{|l|}{ DNA Viruses } \\
\hline Varicella-zoster virus & 17 & 0 & 4 & 21 \\
\hline Herpes simplex virus 1 & 12 & 0 & 1 & 13 \\
\hline Epstein-Barr virus & 12 & 0 & 3 & 6 \\
\hline Cytomegalovirus & 4 & 0 & 0 & 2 \\
\hline Herpes simplex virus 2 & 2 & 0 & 0 & 2 \\
\hline Suid herpesvirus 1 & 2 & 0 & 0 & 2 \\
\hline BK polyomavirus & 1 & 0 & 0 & 0 \\
\hline John Cunningham virus & 1 & 0 & 0 & 1 \\
\hline \multicolumn{5}{|l|}{ Fungi } \\
\hline Cryptococcus neoformans & 7 & 0 & 3 & 10 \\
\hline Cryptococcus gattii & 1 & 0 & 0 & 1 \\
\hline \multicolumn{5}{|l|}{ Parasites } \\
\hline Taenia solium & 5 & 0 & 0 & 5 \\
\hline Angiostrongylus cantonensis & 1 & 0 & 0 & 1 \\
\hline Totals & 110 & 12 & 16 & 114 \\
\hline
\end{tabular}

${ }^{*}$ TP: true-positive; FP: false-positive; FN: false-negative; mNGS: metagenomic

573 next-generation sequencing; CSF: cerebrospinal fluid; CNS: central nervous system 


\section{$574 \quad$ Figure legends}

575 Figure 1. Flowchart of patient enrolment and exclusion.

576

577 Figure 2. Distribution of causative pathogens in patients with suspected CNS

578 infections initially detected by NGS of CSF. (A) Of the $36.6 \%$ patients first diagnosed

579 with NGS of CSF, $16.3 \%$ were diagnosed with bacterial infections, $15.2 \%$ with viral

580 infections, $2.9 \%$ with fungal infections, and $2.2 \%$ with parasitic infections. (B) NGS

581 detected 11 bacterial species, the most common of which were M. tuberculosis

582 (13.9\%) and L. monocytogenes (7.9\%), 7 viral species, the most common of which

583 were VZV (16.8\%) and HSV1 (11.9\%), 2 fungal species, both of which were

584 Cryptococcus $(7.9 \%)$ species, and 2 parasitic species, the most common of which was

585 T. $\operatorname{solium}(5.0 \%)$. 


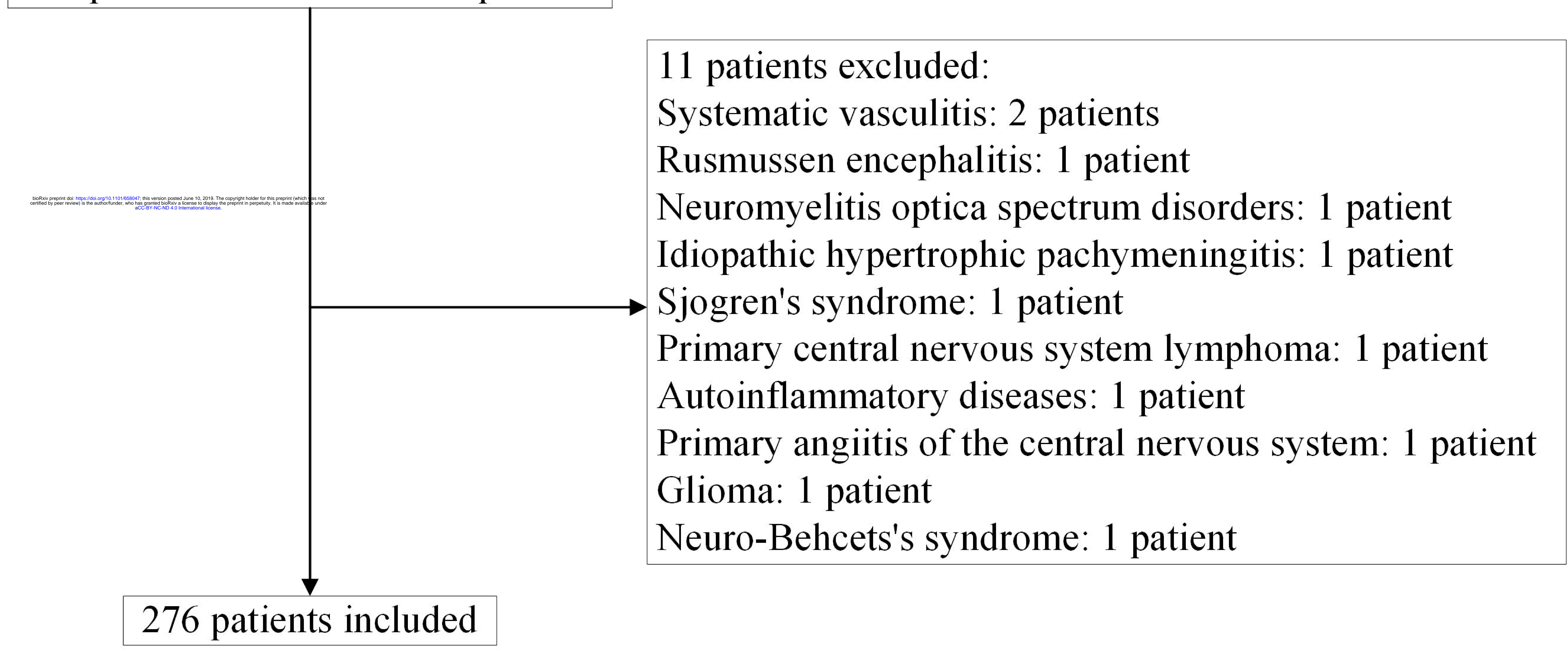


B
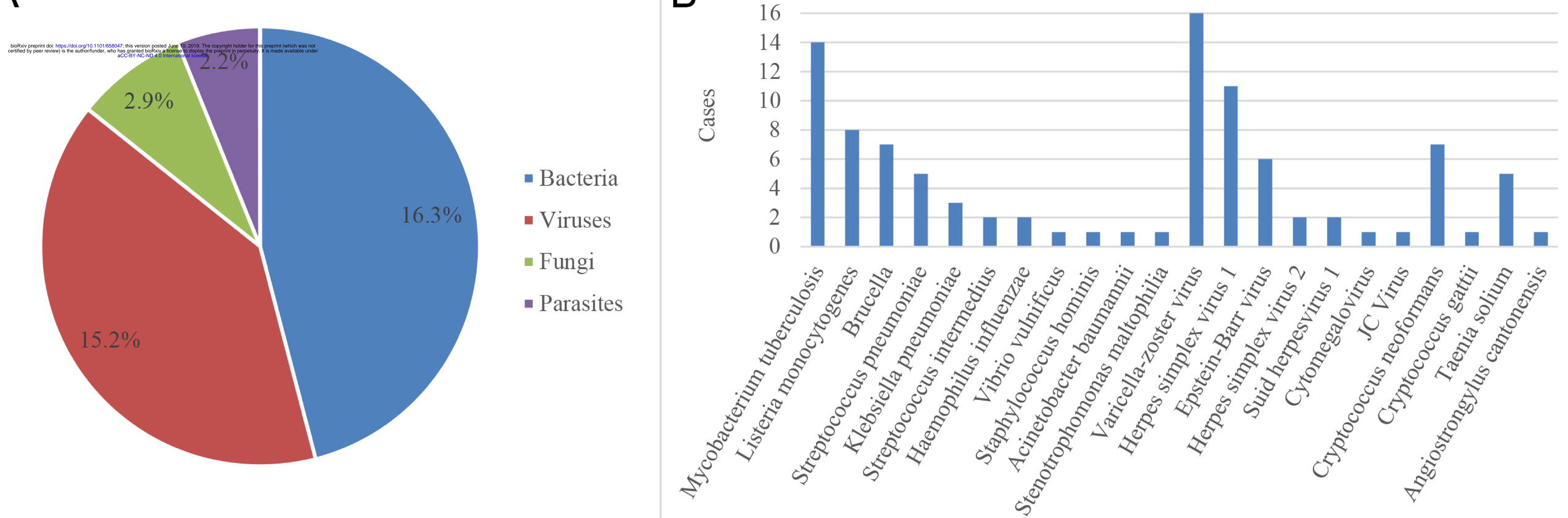